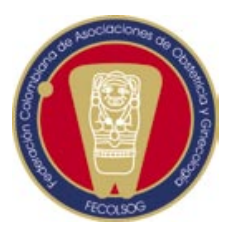

Artículo de Revisión

\title{
VISIÓN ACTUAL DE LA TERAPIA DE SUSTITUCIÓN HORMONAL EN LA MUJER POSMENOPÁUSICA
}

\author{
Current view of the hormone replacement therapy in the \\ postmenopausal woman.
}

John Jairo Zuleta, M.D*

Recibido: noviembre 2/2004. Revisado: diciembre 16/2004. Aceptado: diciembre 20/2004

\section{RESUMEN}

Durante casi una década se esperaron los resultados del estudio WHI (Women's Health Initiative) que se suponía, brindarían información definitiva respecto al empleo de la terapia de reemplazo hormonal en la mujer menopáusica. Para sorpresa de la mayoría, a partir de julio de 2002 han aparecido informes de este estudio, que en general son contrarios a lo que se esperaba y a las creencias que se tenían previamente con respecto a los beneficios y a los riesgos de estas terapias. Las críticas y las posibles explicaciones a estos resultados no se hicieron esperar, y hoy existe bastante confusión al respecto, no sólo para los profesionales de la salud sino especialmente para las mujeres. En el presente artículo se hace una revisión de los aspectos metodológicos más relevantes del estudio WHI y se hace un análisis de las posibles explicaciones para la resistencia a aceptar sus resultados. Se concluye que el estudio tiene validez interna, sus resultados son importantes y la posibilidad de extrapolarlos es bastante alta; también, que en el momento, la única indicación aceptable para la terapia de sustitución hormonal es el control de

* Ginecoobstetra. Magister en Epidemiología Clínica. Profesor asistente del Departamento de Obstetricia y Ginecología y de la Escuela de Investigaciones Médicas de la Facultad de Medicina, Universidad de Antioquia. Calle 37B Sur 27E - 90 Apto 9836, Envigado, Antioquia. Correo electrónico: jjzuleta@epm.net.co la sintomatología vasomotora. Es necesario esperar otros reportes antes de aceptar como terapia de reemplazo otros preparados similares.

Palabras clave: postmenopausia, terapia de reemplazo hormonal.

\section{SUMMARY}

For most part of the last decade the results of the WHI (Women's Health Initiative) were expected with the purpose of obtaining more conclusive information about the uses of Hormonal Replacement Therapy in the postmenopausal patients. But for most people's surprise since july 2002 different reports based on that study have appeared in the medical literature with results different than expected to the prior beliefs we had concerning the risks and benefits to these therapies. The criticism and possible explanations to these results did not wait and were manifested almost immediately and all we have left today is a confusion of big proportions to this respect not only for healthcare professionals but most important for the patients. In this present article a revision of the relevant statistic methods employed is made as well as an analysis of the probable explanations of the people's reluctance to accept the results. It is concluded that the study has an internal validity, its results are important and the possibility to compare them with other situations is high. It is also 
concluded that for the moment the only acceptable indication for Hormonal Replacement Therapy is in the control of the vasomotor symptoms and that it is necessary to wait for the results of future studies before accepting other similar therapies as a valid option for the patient.

Key words: hormone replacement therapy, postmenopause

\section{VISIÓN ACTUAL}

Los ensayos aleatorizados controlados son el patrón de oro de los estudios científicos sobre los efectos del tratamiento. ${ }^{1}$ En ellos el investigador impone las condiciones de selección de individuos, el tratamiento a evaluar, el seguimiento, la forma de medir los desenlaces y planea los análisis desde antes de iniciar la investigación, con el fin de obtener unos resultados libres de sesgos que hagan que si fue bien diseñado, conducido y analizado, las conclusiones obtenidas sean altamente confiables. El ensayo clínico intenta superar los errores sistemáticos o sesgos que se sabe son inherentes a los estudios observacionales, es decir, los estudios descriptivos, de casos y controles y de cohortes. El principal limitante para la validez de estos últimos estudios es que muy frecuentemente existen diferencias entre los grupos comparados y es probable que sean estas diferencias las que expliquen los resultados y no el tratamiento en estudio.

A pesar del auge de la terapia de sustitución hormonal, respaldado por un inmenso número de estudios observacionales, muchos autores estaban convencidos de que aún faltaba solidez en la información para respaldar la implementación masiva de la terapia para prevenir enfermedades crónicas en la mujer y consideraban que mucho del beneficio era explicado por los sesgos de la investigación observacional. ${ }^{2-9}$ Un buen número de los artículos de los años noventa terminaban sus comentarios aclarando que era prudente esperar los resultados de dos grandes ensayos clínicos que se habían iniciado con el fin de resolver definitivamente las preguntas aún vigentes: El Women's Health Initiative (WHI) ${ }^{10,11}$ en Estados Unidos y el Women's International Study of Long Duration Strogen After Menopause (WINSDOM) en el Reino Unido.

\section{EL WHI}

Este estudio es patrocinado por los Institutos $\mathrm{Na}-$ cionales de Salud de los Estados Unidos (NIH) e hizo necesario que se desviaran recursos oficiales de otras investigaciones, después de amplios debates en los organismos del Estado. Incluye un poco más de 161.000 mujeres en 40 centros de todo el país y tiene como objetivo principal evaluar las estrategias para la prevención de la enfermedad cardiovascular, el cáncer de colon y de mama y la osteoporosis, debido a que son las enfermedades que más causan morbimortalidad en las mujeres mayores de todas las razas y estratos socioeconómicos de este país. La iniciativa incluye un estudio observacional que evalúa el estilo de vida y cuatro ensayos clínicos controlados, que evalúan: Estrógenos Conjugados Equinos (ECE) en forma conjunta con Acetato de Medroxiprogesterona (AMP), ECE solos, vitamina $\mathrm{D}$ con calcio y modificación de la dieta. Adicionalmente a los objetivos principales, incluye muchos desenlaces como otros tipos de cáncer, demencia, el compromiso cognitivo e incluso estudios genéticos. Los dos componentes hormonales se debieron suspender antes de la fecha inicialmente planeada porque en ambos se demostró que los riesgos superaban los beneficios que se estaban obteniendo. ${ }^{12,13}$ La suspensión de la rama que estaba evaluando la combinación de estrógeno más medroxiprogesterona influyó para que la contraparte inglesa, el WINSDOM, igualmente se cancelara en forma prematura. ${ }^{14}$

A pesar de que las esperanzas de obtener información concluyente con respecto al real papel de las terapias de sustitución hormonal estaban puestas en los resultados de estos estudios, cuando las conclusiones no fueron tan favorables como se esperaban y muchas de ellas contradecían lo que dicho previamente, empezaron a aparecer una serie de críticas que nunca se le habían hecho, a pesar de que 
el protocolo fue de conocimiento público durante la implementación de los proyectos. ${ }^{9}$ La pregunta que surge es: ¿porqué antes de conocer los resultados, el WHI era considerado el estudio que iba a dar la respuesta definitiva, y ahora que se conocieron sus resultados, aparecen tantos cuestionamientos en su contra? Se podrían proponer cuatro explicaciones a esta situación:

- Realmente existen fallas en el diseño y la implementación de los estudios que invalidan los resultados.

- Aunque sea un estudio válido, sus conclusiones no son extrapolables a otras poblaciones.

- Existen intereses económicos que se ven afectados con los resultados de los estudios y es necesario demeritarlos.

- Las autoridades del tema han dedicado muchos años de su vida académica a defender unas ideas falsas y ahora no les es fácil reconocer el error.

\section{VALIDEZ INTERNA}

\section{DE LOS ESTUDIOS}

Tanto la rama de terapia combinada como la rama de sólo estrógenos cumplen con los criterios para considerar que los resultados realmente son explicados por el efecto de la terapia hormonal y no por sesgos. ${ }^{15}$ Se hizo un proceso de aleatorización que es un mecanismo que garantiza que el ingreso del individuo a alguno de los grupos de estudio no esté definida por alguna de sus características previas, por lo tanto se obtienen dos grupos de comparación con condiciones clínicas y sociales muy similares, tal como se puede confirmar cuando se evalúan las características de las mujeres en los estudios, donde se aprecia que características como edad, índice de masa corporal, presión arterial, entre otras, los antecedentes de enfermedades y la presencia de factores de riesgo para ellas son muy similares entre el grupo que tomó el medicamento y el respectivo control que recibió placebo.

El enmascaramiento de la asignación es un elemento tanto o más importante que la misma aleatorización. Con ello se busca que la persona que esté asignando el grupo en que se va a incluir al individuo, no sea capaz de predecir y por lo tanto pierda la posibilidad de manipular a conveniencia tal asignación. La única forma de identificar al grupo era mediante un código de barras en el frasco que contenía los tratamientos, cuya lectura era posible únicamente en la oficina central.

Los estudios respetaron el enmascaramiento tanto de los investigadores, como de los médicos tratantes y las mujeres, y con esto se impide que al conocer el grupo al que pertenecen los individuos en un estudio, se implementen en forma diferencial otras medidas diagnósticas o terapéuticas que modifiquen el pronóstico de alguno de los grupos. En el WHI todas las mujeres son sometidas a un protocolo de control y seguimiento idéntico, excluyendo que las cointervenciones afecten favorablemente a alguno de los grupos, tal como sucedía en los estudios observacionales. En el estudio de estrógeno más progestina, el ginecólogo tratante, pero nunca los investigadores, supo el grupo de asignación en un $24 \%$ y en el de solo estrógeno un $1,7 \%$.

Una de las grandes dificultades de los estudios con seres humanos es que no se puede garantizar que todos los individuos cumplan en forma estricta y completa todo lo propuesto en un protocolo. Los individuos tienen la potestad de retirarse o cambiar de intervención. Los estudios del WHI no son la excepción a esto y en ellos se presentó en una proporción muy importante: del estudio de estrógeno más progestina, $42 \%$ se retiraron del grupo de medicación y $38 \%$ del grupo de placebo, en la rama de sólo estrógeno estas proporciones fueron de 53,8\% para cada grupo. Esta situación es una de las más conflictivas que tienen que resolver quienes analizan los estudios. El recurso metodológico más aceptado hasta el momento es el conocido como análisis por intención de $\operatorname{tratar}^{16}$ que consiste en asignar los desenlaces al grupo en el cual fueron aleatorizados los individuos, independiente de que hayan cumplido o no con la medicación que les correspondía. A pesar de lo antiintuitivo, se ha demostrado que este análisis 
impide que se sobrevalore la real eficacia de una intervención. Cuando se hace este tipo de análisis para resolver la falta de adherencia a los tratamientos, se sabe que si los individuos hubieran cumplido completamente con el protocolo, tanto los efectos benéficos como los dañinos serían superiores. Los resultados del WHI se presentan con este tipo de análisis. Las otras alternativas serían excluir de los análisis a los individuos que no cumplieron, conocido como análisis según tratamiento, o incluirlos en el grupo en el que realmente estuvieron, análisis por protocolo. En el WHI se hicieron ambos análisis y se confirmó que no cambiaba la dirección de los resultados y que efectivamente aumentan los valores de los riesgos medidos.

Un aspecto que disminuye la validez en los estudios son las pérdidas del seguimiento. En la rama de terapia combinada se tiene información del 96,5\% de las mujeres y en el de solo estrógeno del 94,8\%, cifras de seguimiento por debajo de las pérdidas aceptadas que son del 10 a $20 \%$.

Teniendo en cuenta estos aspectos, se pueden considerar como estudios válidos, por lo tanto sus resultados son creíbles; se puede asegurar que las diferencias encontradas entre los grupos son debidas a lo único que los diferencia: recibir o no la terapia hormonal. Si se acepta que los métodos empleados fueron válidos, tiene sentido analizar los resultados.

\section{RESULTADOS}

La tabla 1 resume los resultados encontrados en ambos estudios, se presenta el número de eventos en cada uno de los grupos y el Hazard Ratio que es una medida de asociación que cuantifica el riesgo, pero teniendo en cuenta el tiempo que cada persona estuvo expuesta, con su respectivo intervalo de confianza del 95\%. Los autores presentaron tanto intervalos nominales como ajustados, acá se presentan los nominales debido a que son los que ellos emplean para las conclusiones, porque aducen que son los que permiten comparar con otros estudios que siempre han utilizado este tipo de intervalo que no ajusta por las múltiples comparaciones, que es el objetivo del ajustado. ${ }^{12}$

En resumen, la combinación de 0,65 mg de ECE con 2,5 mg de AMP en forma continua aumenta el riesgo de sufrir enfermedad coronaria, enfermedad cerebrovascular, enfermedad trombótica y cáncer de mama y disminuye la posibilidad de sufrir fracturas o de padecer de cáncer de colon. El empleo de igual dosis de ECE sin progestina aumenta el riesgo de sufrir enfermedad cerebrovascular, disminuye el de sufrir fracturas y no parece ejercer efecto ni benéfico ni deletéreo sobre la enfermedad coronaria, la trombosis, el cáncer de mama o colon. Este balance general de riesgos y beneficios es el que llevó a los investigadores a concluir que no se recomiendan estas terapias para la prevención de enfermedades crónicas de la mujer posmenopáusica.

\section{EXTRAPOLACIÓN DE LOS ESTUIDIOS}

A los estudios se les evalúan dos tipos de validez: interna y externa. La interna se refiere a si los autores seleccionaron el modelo más adecuado para responder la pregunta y si se emplearon en forma rigurosa los métodos para implementarlo. La externa es la que permite que los resultados de la investigación sean aplicados a otros individuos. Las críticas que se le hacen al WHI se encuentran principalmente en este aspecto: se aduce que se empleó un grupo de mujeres con edad superior a la que tienen las mujeres a las cuales se les está recomendando la terapia en la vida diaria, que son mujeres obesas, que no son realmente sanas, que no son representativas de las mujeres de nuestro país, que no se emplearon otras vías de administración u otros compuestos. Para resolver si los resultados de una investigación son aplicables a un paciente en particular lo que se busca es identificar si esta persona hubiera sido candidata a ser incluida en el trabajo.

Aunque el promedio de edad de las mujeres en esta investigación fue de 63,3 y 63,6 años, es claro que hubo representación de mujeres desde los 50 hasta los 79 años. La distribución por grupos de edad se estableció desde que se diseñó el 
Tabla 1. Principales resultados de los estudios de terapia de sustitución hormonal en el WHI

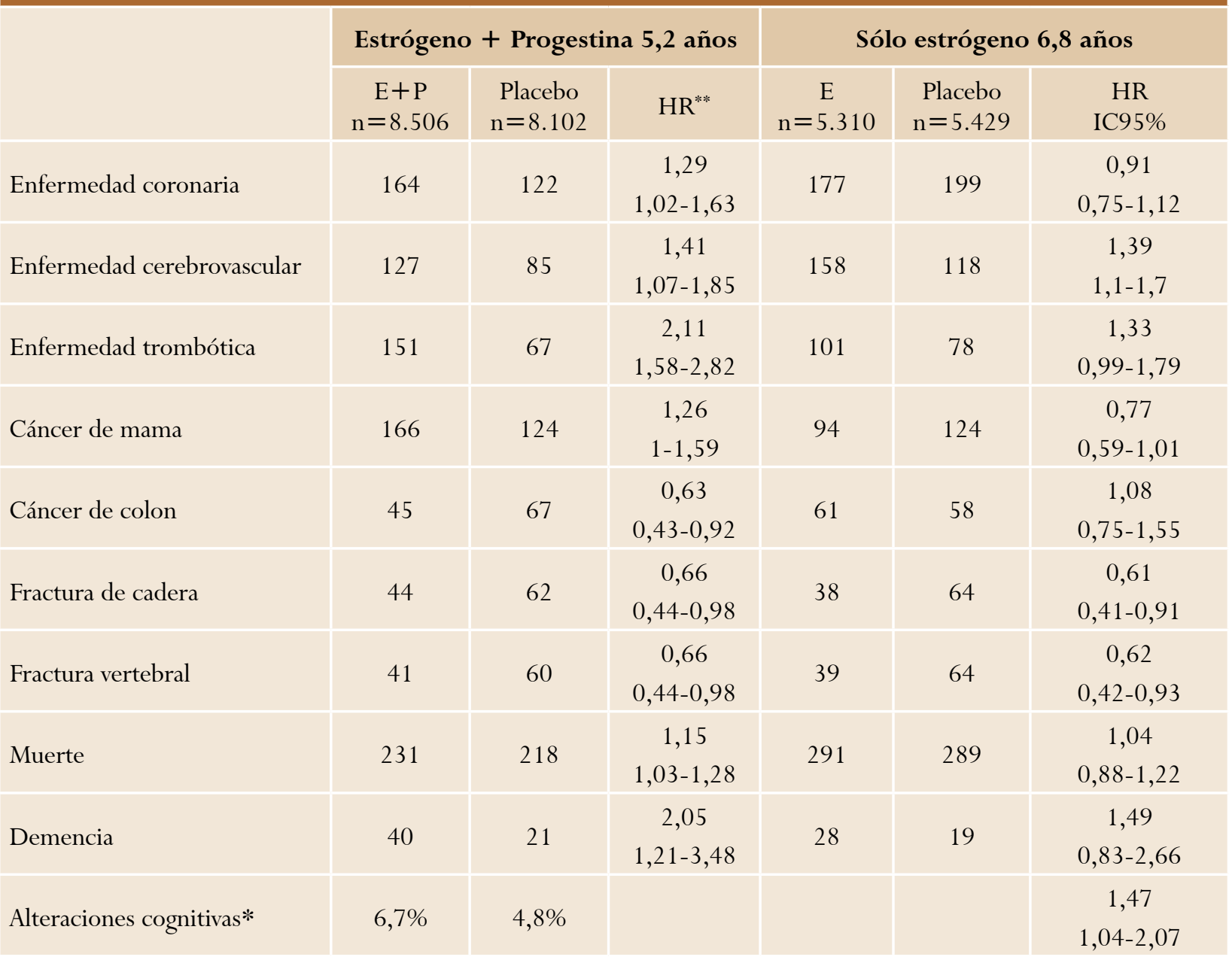

Editados de referencias 12, 13, 44, 45, 46, 47.

* Estos resultados se basan en un subgrupo seleccionado para participar en el Womens Health Initiative Memory Study: 4.532 para la rama que evaluó la terapia combinada y 2.808 para la rama con sólo estrógeno. Los resultados se presentaron de maneras diferentes en los informes.

** El HR presenta el riesgo de disminuir 10 unidades en el test minimental.

protocolo y es consecuencia de un análisis juicioso previo que buscaba optimizar los recursos, incluyendo un número representativo en cada grupo que permitiera tener suficiente número de desenlaces deseados. Tuvieron en cuenta que la presentación de las enfermedades que se querían estudiar era diferente según la edad, por lo tanto era necesario tener un rango amplio. Para el momento de inicio, contrario a lo que se dice hoy, se cuestionó que se incluyeran mujeres menores de 55 años. El protocolo estableció un $10 \%$ de mujeres entre 50 y 54, un $20 \%$ entre 55 y 59 , un $45 \%$ entre 60 y 69 y un $25 \%$ entre 70 y 79 años. El raciocinio fue que si se incluían muchas más mujeres entre 50 y 55 años se reduciría notablemente el poder, debido a que la incidencia de enfermedad coronaria aumenta con la edad y se distorsionaría el perfil de riesgo beneficio, porque la relación entre la incidencia de cáncer de mama y de enfermedad coronaria es más alto en las mujeres posmenopáusicas jóvenes que entre las mayores. ${ }^{10}$ Como se proponía valorar el efecto sobre la enfermedad mental, era necesario incluir 
un grupo de mujeres añosas que es en quienes más frecuentemente se presenta el problema.

Al momento de cuestionar la influencia de la edad y otras de las variables consideradas problemáticas, es necesario tener claridad con el concepto estadístico conocido como ajuste de variables de confusión. ${ }^{17}$ En condiciones multifactoriales como la enfermedad cardiovascular, la osteoporosis o el cáncer, es obvio que son muchos los factores que influyen y modifican su aparición. El recurso metodológico para controlar todas estas influencias y asignar el peso que tienen en determinada población es el análisis multivariante. El análisis de riesgos proporcionales de $\mathrm{Cox}^{18}$ empleado en estas investigaciones es uno de ellos, que adicionalmente tiene la virtud de permitir controlar la influencia que tiene el tiempo de uso de la medicación en una situación donde las mujeres la emplearon por períodos distintos. Cuando se presentan los resultados, se aclara que fueron ajustados y se enumera por cuales características. Esto significa que se hicieron comparaciones en igualdad de condiciones, por ejemplo, al ajustar por hábito de cigarrillo quiere decir que se compara el grupo de fumadoras tomando hormona, con el grupo de fumadoras tomando placebo, y se comparan igualmente las no fumadoras de ambos grupos entre sí, para evaluar si los resultados se modifican o persisten. En general, los resultados encontrados confirman que las hormonas ejercieron su influencia independiente, tanto en los efectos benéficos, como en los dañinos. En las ampliaciones del informe original se puede confirmar esto. La tabla 2 muestra el efecto de la combinación estrógeno más progestina en la enfermedad coronaria según grupos de edad, niveles de índice de masa corporal, antecedente de hipertensión o enfermedad cardiovascular. ${ }^{19}$ Es de aclarar que en estos análisis de subgrupos al fraccionar la muestra se pierde el poder de encontrar

Tabla 2. Influencia de las diferentes categorías de algunas de las características en la aparición de enfermedad coronaria en mujeres del estudio de estrógeno más progestina del WHI

\begin{tabular}{|c|c|c|c|c|}
\hline & $\begin{array}{c}\text { Estrógeno }+ \\
\text { Progestina } \\
\text { n (\% por año) }\end{array}$ & $\begin{array}{c}\text { Placebo } \\
\text { n (\% por año) }\end{array}$ & HR & $\frac{\mathrm{p}}{\text { de interacción }}$ \\
\hline \multicolumn{5}{|c|}{ Edad } \\
\hline 50-59 años & $37(0,22)$ & $27(0,17)$ & 1,27 & \multirow{3}{*}{0,36} \\
\hline 60-69 años & $75(0,35)$ & $68(0,34)$ & 1,05 & \\
\hline 70-70 años & $76(0,78)$ & $52(0,55)$ & 1,44 & \\
\hline \multicolumn{5}{|c|}{ Índice de masa corporal } \\
\hline$<25 \mathrm{~kg} / \mathrm{m}^{2}$ & $53(0,36)$ & $37(0,27)$ & 1,38 & \multirow{3}{*}{0,6} \\
\hline $25-29,9 \mathrm{~kg} / \mathrm{m}^{2}$ & $64(0,38)$ & $53(0,33)$ & $1, .23$ & \\
\hline$>30 \mathrm{~kg} / \mathrm{m}^{2}$ & $71(0,44)$ & $57(0,38)$ & 1,16 & \\
\hline \multicolumn{5}{|c|}{ Antecedente de hipertensión arterial } \\
\hline No & $81(0,26)$ & $66(0,23)$ & 1,14 & 0,49 \\
\hline $\mathrm{Si}$ & $107(0,65)$ & $81(0,50)$ & 1,32 & \\
\hline \multicolumn{5}{|c|}{ Antecedente de enfermedad cardiovascular } \\
\hline No & $156(0,34)$ & $118(0,28)$ & 1,23 & 0,64 \\
\hline $\mathrm{Si}$ & $29(1,64)$ & $24(1,19)$ & 1,45 & \\
\hline
\end{tabular}

Editado de la referencia 19. 
asociaciones estadísticamente significativas porque el cálculo de tamaño de muestra no se hizo para el subgrupo sino para toda la población. Esto explica la ausencia de significancia estadística si se evalúan los intervalos de confianza o si se hicieran las pruebas estadísticas pertinentes. Se aprecia que los valores de p que evalúan la interacción del medicamento con la característica que se estudia no son significativos, lo que descarta que el efecto obtenido se deba a esas características y confirma que independiente de los diferentes valores de ellas, la utilización de la combinación hormonal ejerce su efecto.

En cuanto al índice de masa corporal, es claro que la distribución encontrada con una alta proporción de mujeres con sobrepeso y obesas es un reflejo de las características normales de la población norteamericana, y el estudio se diseñó para dar soluciones a esa población. Vale la pena resaltar que ni el sobrepeso ni la obesidad fueron nunca contraindicaciones para el empleo de la terapia de sustitución hormonal e igualmente en los estudios observacionales que dieron sustento a la propuesta del efecto benéfico de la terapia había un buen número de ellas. Como se puede ver en la tabla 2 , el índice de masa corporal no modificó los resultados y los efectos de la terapia no se modificaron para ningún nivel de él.

El trabajo fue planteado para evaluar una práctica que ya era rutinaria. La terapia se empleaba para disminuir el riesgo de enfermedad coronaria, y la hipertensión controlada u otras enfermedades cardiovasculares no eran contraindicación para su uso, de hecho esta población fue objeto de un ensayo clínico controlado, el estudio HERS ${ }^{20}$ que intentaba confirmar la creencia de que la terapia hormonal de sustitución en la posmenopausia era una buena alternativa de prevención secundaria. Algunos proponen descartar el estudio por la inclusión de mujeres con enfermedades al inicio: lo que se debe hacer es confirmar que ellas estén distribuidas por igual proporción en ambos grupos de comparación y evaluar qué resultados tuvieron. Nuevamente la tabla 2 demuestra que el efecto deletéreo de la terapia se presentó independiente de la presencia o no de estas enfermedades. Es de resaltar que el WHI confirmó los efectos benéficos de la terapia en el perfil lipídico, pero esto no tuvo efecto sobre lo importante que es la aparición de la enfermedad. ${ }^{19} \mathrm{Con}$ esto se confirma el peligro de tomar decisiones con los llamados desenlaces intermedios o subrogados: lo importante no es que se mejoren los marcadores bioquímicos sino que eso se refleje en un resultado importante para las personas.

Los informes que amplían el efecto sobre las fracturas, ${ }^{21}$ el cáncer de mama ${ }^{22}$ y el cáncer de colon $^{23}$ confirman la independencia de los efectos tanto benéficos como dañinos de la combinación ECE - AMP, porque igualmente en ellos se muestran los resultados según diferentes categorías de edad, índice de masa corporal, antecedentes familiares y personales, tiempo de evolución de la menopausia antes del inicio de la terapia, consumo de cigarrillo, presencia de factores de riesgo para enfermedades específicas, entre otros. Vale la pena resaltar la interpretación de estos hallazgos cuando se hacen llamados para individualizar la administración según los factores de riesgo: la terapia hormonal ejerce su efecto independiente de que la mujer tenga o no tenga factores de riesgo adicionales.

Con respecto a la raza, es llamativo que antes del WHI nunca se cuestionó si la información obtenida en los estudios observacionales, realizada en población típica norteamericana o europea era extrapolable a nuestra población latina. La mayoría de las investigaciones se hacen con población diferente a la nuestra y esto no ha sido freno para aplicar los resultados: en el estudio MORE para evaluar el raloxifeno el 95,7\% de las mujeres eran blancas, ${ }^{24} \mathrm{el}$ estudio GUSTO para evaluar la terapia trombolítica después del infarto fue realizado en Norte América, Europa, Israel, Nueva Zelanda y Australia y no menciona que haya habido latinos en su población, ${ }^{25} \mathrm{el}$ estudio CLASS, uno de los pioneros en el estudio de los antiinflamatorios inhibidores de la COX 2, tuvo únicamente un 2,7\% de hispanos en cada grupo de comparación. ${ }^{26}$ El estudio de cohorte más grande que evalúa la TRH, el Estudio de las Enfermeras, 
inicialmente no consideró la raza de sus participantes e incluyó esta información únicamente 6 años después de iniciado ${ }^{27}$ y es excepcional que la describa en sus informes. ${ }^{28-31}$ Estudios actuales con respecto al efecto de la terapia de sustitución continúan empleando casi exclusivamente mujeres blancas, 81 a $97 \%,{ }^{32,33}$ no describen la raza o no individualizan el grupo de hispanas, las cuales quedan dentro de un grupo llamado "otras" 33 e incluso excluyen a las que hablan español. ${ }^{34}$ Por último, no existe descripción de medicamentos que tengan una acción en una raza y el opuesto en otra. Nuevamente, la selección de las etnias para los estudios del WHI fue intencional: se incluyó una proporción de mujeres negras, hispanas, asiáticas o de origen indígena que reflejara la distribución propia de los Estados Unidos. ${ }^{10} \mathrm{El}$ WHI tiene mayor cantidad de mujeres diferentes a las blancas de las que tienen la mayoría de estudios en terapia de sustitución hormonal.

Con respecto a las otras vías de administración, las otras dosis, los otros esquemas de administración $\mathrm{u}$ otros principios activos, los autores del WHI son claros en afirmar que las conclusiones obtenidas de sus estudios no son aplicables a estas otras alternativas. La justificación del empleo del esquema evaluado es que esta era la combinación más frecuentemente recomendada en los Estados Unidos y sobre la cual existía más respaldo bibliográfico y la forma continua de administrarla, porque era la alternativa que permitía mantener el enmascaramiento, porque no inducía los sangrados cíclicos. ${ }^{10}$ Es claro que aunque estos hallazgos no son extrapolables a esas presentaciones, tampoco existen en este momento investigaciones con la solidez del WHI para descartar que no tengan los mismos resultados.

\section{INTERÉS DE}

\section{LA EMPRESA FARMACÉUTICA}

En la literatura desde hace bastante tiempo están bien documentadas las estrategias de la empresa farmacéutica para generar la necesidad de iniciar tratamientos induciendo la asimilación de ciertos factores de riesgo o condiciones fisiológicas como enfermedad, ${ }^{35}$ la influencia sobre los patrones de recomendaciones terapéuticas de los médicos, ${ }^{36}$ las estrategias de convencimiento ${ }^{37,38}$ o de comunicación ${ }^{39}$ e incluso la manipulación antiética de la información. ${ }^{40}$

Pocos meses después de que se conocieron los resultados de la primera rama del estudio WHI, salieron a la luz pública dos informes de intentos de manipular la información por parte de dos de las principales compañías involucradas en la investigación y comercialización de preparados hormonales. En el primero, ${ }^{41}$ se informó que un laboratorio distribuyó información confidencial aún no publicada sobre el efecto deletéreo de la terapia sobre la demencia con el fin de preparar la defensa posterior. En el segundo informe, ${ }^{42}$ se acusó a otra multinacional de haber empleado el nombre del presidente de las organizaciones alemanas de profesionales para distribuir un documento a miles de ginecólogos demeritando los hallazgos de las investigaciones del WHI.

Una de las estrategias preferidas en los diferentes impresos recibidos ha sido menospreciar los efectos dañinos, insistiendo en que los resultados presentados en términos relativos son más impresionantes que lo que realmente se presenta en términos absolutos. Esto es verdad, pero es igualmente válido para los efectos benéficos. La combinación ECE - AMP demostró aumentar en un 29\% la incidencia de enfermedad cardiovascular y disminuir en un $24 \%$ el riesgo de cualquier fractura secundaria a osteoporosis. Estos valores se obtienen después de encontrar que de 8.102 mujeres que no tomaron las hormonas durante un promedio de 5,2 años, 122 $(1,51 \%)$ tuvieron algún evento cardiovascular pero de 8.506 que las tomaron, 164 (1,93\%) presentaron igual desenlace. Obviamente en términos absolutos se aprecia que el riesgo es bajo. Con respecto a las fracturas, al cabo de 5,2 años, de 10.000 mujeres que empleen hormona, solamente 44 (0,52\%) presentarían una fractura de cadera, pero si no la toman, $62(0,77 \%)$ sí la presentarían, lo cual también como número absoluto es bajo. 
Ante los primeros resultados publicados del WHI, se llamó la atención acerca de la ausencia de información acerca de la calidad de vida y las funciones mentales. Posteriormente se demostró que no tenían efecto sobre la calidad de vida ${ }^{43}$ y que aumentaban el riesgo de demencia. ${ }^{44,45,46,47}$ Un informe reciente del Estudio de las Enfermeras en el cual realizaron los ajustes por variables de confusión que previamente no habían tenido en cuenta, igualmente encontró un mayor deterioro en las funciones mentales de las mujeres que emplearon TRH. ${ }^{48}$ Es importante resaltar que el concepto de calidad de vida va mucho más allá de la presencia o la ausencia de unos síntomas molestos para los individuos. En el WHI, para valorar la calidad de vida ${ }^{43}$ se midió el estado funcional de las mujeres, que tuvo en cuenta la salud general, el funcionamiento físico, las limitaciones en las actividades rutinarias, el dolor corporal, la energía y la fatiga, los problemas emocionales o mentales, el funcionamiento social y la salud mental o emocional e igualmente se evaluó la depresión, las alteraciones del sueño, el desempeño sexual, el funcionamiento cognitivo y los síntomas. Al evaluar la TRH en forma grupal es obvio encontrar que el grupo que presenta más casos de cáncer de mama, de enfermedad trombótica, de enfermedad coronaria, enfermedad cerebrovascular, de sangrado genital anormal, de histerectomías y de colecistectomías ${ }^{12,13}$ tiene menor calidad de vida.

Zuckerman, ${ }^{49}$ llamando la atención sobre la distorsión de las noticias médicas en los periódicos, presenta las estrategias de la empresa farmacéutica para convencer a los medios de comunicación y a las usuarias de los beneficios que ya la investigación había descartado. Igualmente resalta cómo después de 20 años de publicado el texto que más influyó en las decisiones de la mujer para acoger la TRH, "Femenine for ever" de Robert Wilson, se descubrió que este libro había sido pagado por una empresa farmacéutica con bastantes intereses en el tema.

\section{OPINIÓN DE LOS EXPERTOS}

Meier Stampfer en compañía de Graham Colditz, coinvestigadores del estudio de cohortes más grande en TRH, el Estudio de la Salud de las Enfermeras, presentaron en el año 1991 uno de los metanálisis que respaldaba el empleo de la TRH para disminuir el riesgo de enfermedad coronaria, ${ }^{50}$ y en él consideraron remota la posibilidad de que los hallazgos fueran explicados por posibles variables de confusión no controladas en los estudios en los que basó su análisis. Hoy Stampfer, plantea que aunque es probable que haya diferencias de resultados entre el WHI y los estudios observacionales que él analizó previamente, si se lograra demostrar que la TRH es benéfica para las mujeres más jóvenes, sería inaceptable recomendarla porque de todas maneras el balance de riesgos y beneficios permanecería desfavorable para la TRH, más teniendo en cuenta que existen alternativas más seguras. ${ }^{46} \mathrm{Su}$ compañero de publicación, Graham Colditz, escribió en compañía de Hellen Hulley el editorial de la revista JAMA que acompañó el informe inicial de los resultados del WHI el 17 de julio de 2002. En él, aunque expresa su extrañeza con los resultados y acepta que los riesgos individuales son pequeños, reconoce que los riesgos superan los beneficios y recomienda que las mujeres que estén empleando la TRH con la intención de prevenir enfermedades crónicas, la suspendan. ${ }^{52}$

La doctora Débora Grady generó un metanálisis con un título bastante provocativo apoyando el empleo de la TRH: "Terapia hormonal para prevenir enfermedad y prolongar la vida en mujeres posmenopáusicas". ${ }^{33}$ En él se sugirió que la TRH podría ser beneficiosa para mujeres con enfermedad coronaria o con riesgo de ella y que para otras mujeres la situación no era aun clara. Después de participar en la implementación de las ramas de tratamiento hormonal del WHI, generó un editorial en el New England Journal of Medicine con un título igualmente impactante: "Hormonas en la menopausia, terapia únicamente para síntomas". En este escrito plantea una pregunta de máxima vigencia: aunque los riesgos absolutos del empleo de la TRH son muy pequeños, "porqué los deben asumir las mujeres?. Concluye que la TRH no tiene utilidad en mujeres 
asintomáticas, que las sintomáticas deberían sopesar los riesgos y los beneficios antes de tomar la decisión de emplearla y que se deberían buscar tratamientos igualmente efectivos para la sintomatología menopáusica pero más seguros. ${ }^{54} \mathrm{La}$ doctora Grady fue acompañada en este metanálisis por la doctora Diana Petiti, una investigadora del tema. La doctora Petiti en un comentario reciente igualmente cuestiona la validez de las conclusiones de los estudios previos. ${ }^{55}$ La doctora Grady confirma sus apreciaciones incluso a pesar del aparente efecto menos dañino de los estrógenos solos. ${ }^{56}$

La doctora Francine Grodstein, investigadora principal del Estudio de la Salud de las Enfermeras, igualmente aceptó que aunque todavía falta bastante por aprender, ante la información obtenida a partir del WHI no se debe iniciar o continuar la terapia con el fin de prevenir enfermedad cardiovascular, independiente de la edad de la mujer porque el precio que se paga con el cáncer de mama, el tromboembolismo venoso y la enfermedad cerebrovascular es demasiado alto. ${ }^{57}$

Obviamente, no todas las autoridades del tema han aceptado estas conclusiones y algunos aún proponen que se pueden esperar beneficios en algunos grupos poblacionales específicos. El doctor Frederick Naftolin, ${ }^{58}$ una autoridad académica con amplia experiencia en la investigación básica pero marginal en la investigación clínica, y su grupo, consideran que el estudio no tuvo el suficiente poder para detectar diferencias estadísticamente significativas para las mujeres que realmente se podrían beneficiar de la TRH: las que inician su empleo en edades más tempranas, menores de 55 años, y al poco tiempo de haber llegado a la menopausia. Proponen que con muestras pequeñas, como la del grupo de mujeres de 50 a 54 años que contó únicamente con 574 mujeres, la detección de diferencias en la ocurrencia de eventos infrecuentes es problemática. Es bueno aclarar que aunque esto es verdad, las 5.522 mujeres de este rango de edad incluidas en la rama de terapia combinada y las 3.310 de la rama de sólo estrógeno del WHI, superan ampliamente el número incluido en muchos de los estudios previos que mostraban beneficio.

El doctor Manuel Neves-E-Castro ${ }^{59}$ igualmente propone que este grupo de mujeres aún podría obtener beneficio, aunque reconoce que existen otras alternativas más inocuas para prevenir la enfermedad coronaria. En el Estudio de la Salud de las Enfermeras la incidencia de eventos coronarios en el grupo de 50 a 54 años fue de 53 de 100.000 mujeres por año. Naftolin enfatiza que por lo tanto se requerirían más de 4.000 en un ensayo clínico para poder encontrar diferencias. Aunque esto pudiera ser verdad, es bueno tener en cuenta que si se lograra demostrar una disminución del 50\% en la incidencia en este grupo, sería necesario tratar a 3.774 mujeres para prevenir un solo caso, lo cual no es aceptable teniendo en cuenta los daños confirmados en las otras enfermedades.

\section{CONCLUISIÓN}

Es suficientemente conocida la expresión que el primer objetivo del médico es no hacer daño. Con la información disponible en la actualidad se puede asegurar que no hay nada que respalde el empleo de ECE sólos o en combinación con AMP, para prevenir enfermedades crónicas de las mujeres menopáusicas. Con respecto a otros compuestos, dosis o vías de administración, no existe investigación suficientemente sólida que permita asegurar que no tienen igual comportamiento, por lo tanto no es prudente recomendarlos en reemplazo de los que ya demostraron que no son benéficos: la ausencia de evidencia de daño no es sinónimo de evidencia de ausencia de daño. Por ahora, hasta que no haya nuevas investigaciones de suficiente validez que demuestren lo contrario, la recomendación es hacer caso a la doctora Débora Grady: emplear TRH únicamente para controlar síntomas.

\section{REFERENCIAS}

1. Fletcher RH, Fletcher S, Wagner EH. Epidemiología clínica. Aspectos fundamentales. $2^{\mathrm{a}}$ ed. Barcelona; 1998. 
2. Rossouw JE. Estrogens for prevention of coronary heart disease. Putting the brakes on the bandwagon. Circulation 1996;94:2982-5.

3. Mathews KA, Kuller LH, Wing RR, Meilahn EN, Plantinga P. Prior to use of estrogen replacement therapy, are user healthier than nonusers? Am J Epidemiol 1996;143:971-8

4. Barrett-Connor E. Postmenopausal estrogen and prevention bias. Ann Intern Med 1991;115:455-6.

5. Sturgeon S, Schairer C, Brinton LA, Pearson T, Hoover R. Evidence of healthy estrogen user survivor effect. Epidemiology 1995;6:227-31.

6. Posthuma W, Westendorp RG, Vanderbrouche JP. Cardioprotective effect of hormone replacement therapy in posmenopausal women: is the evidence biased? BMJ 1994;308:1268-9.

7. Grondstein F. Invited comentary: can selection bias explain the cardiovascular benefits of estrogen replacement therapy? Am J Epidemiol 1996;143:979-82.

8. Hemminki E, McPherson K. Impact of postmenopausal hormone therapy on cardiovascular events and cancer: pooled data from clinical trials. BMJ 1997;315:149-53.

9. Zuleta J. Terapia de reemplazo hormonal y enfermedad coronaria: beneficios explicados por los sesgos en la investigación. Rev Colomb Obstet Ginecol 2003;54:113-20.

10. The Women's Health Initiative Group. Design of the Women's Health Initiative Clinical trial and observational study. Controlled Clin Trials 1998;19:61-109.

11. Freedman L, Anderson G, Kipnis V, Prentice R, Wang CY, Rossow J, et al. Approached to monitoring the results of long-term disease prevention trials: examples from the Women's Health Initiative. Controlled Clin Trials 1996; 17:509-25.

12. Rossouw JE, Anderson GL, Prentice RL, LaCroix AZ, Kooperberg C, Stefanick ML, et al; Writing Group for the Women's Health Initiative Investigators. Risk and benefits of estrogen plus progestin in healthy posmenopausal women: principal results From the Women's Health Initiative randomized controlled trial. JAMA 2002;288:321-33.

13. Anderson GL, Limacher M, Assaf AR, Bassford T, Beresford SA, Black H, et al; The Women's Health Initiative Steering Commitee. Effects of conjugated equine estrogen in postmenopausal women with hysterectomy: the Women's Health Initiative randomized controlled trial. JAMA 2004;291:1701-12.

14. White C. Second long term HRT trial sttoped early. BMJ 2002;325:987.
15. Guyatt GH, Sackett DL, Cook DJ. Users' guides to the medical literature. II. How to use an article about therapy or prevention. A. Are the results of the study valid? Evidence-Based Medicine Working Group. JAMA 1993;270:2598-601.

16. Montori VM, Guyatt GH. Intention-to-treat principle. CMAJ 2001;165:1339-41.

17. Kleinbum D, Kupper LL, Morgenstern H. Chapter 13. Confounding. In: Kleinbum D, Kupper L, Morgenstern H. Epidemiologic research: principles and quantitative methods. New York: Van Nostrand Company; 1982. p. 243-65.

18. Kleinbaum D. The Cox Proportional Hazard Model and its caracteristics. In: Kleinbaum D. Survival Analysis. A self-learning text. 1st ed. New York: Springer Verlag; 1996.

19. Manson J, Hsia J, John son K, Rossouw J, Assaf AR, Lasser N, et al; Women's Health Initiative Investigators. Estrogen plus progestin and the risk of coronary heart disease. N Engl J Med 2003;349:523-34.

20. Hulley S, Grady D, Bush T, Furberg C, Herrington D, Riggs B, Vittinghoff E. Randomized trial of estrogen plus progestin for secondary prevention of coronary heart disease in postmenopausal women. JAMA 1998;280:605-13.

21. Cauley JA, Robbins J, Chen Z, Cummings SR, Jackson $\mathrm{RD}$, LaCroix A, et al. Women's Health Initiative Investigators. Effects of estrogen plus progestin on risk of fracture and bone mineral density. The Women's Health Initiative randomized trial. JAMA 2003;290: 1729-38.

22. Chlebowski RT, Hendrix SL, Langer RD, Stefanick ML, Gass M, Lane D, et al; WHI Investigators. Influence of estrogen plus progestin on breast cancer and mammography in healthy posmenopausal women: the Women's Health Initiative Randomized Trial. JAMA 2003;289:3243-53.

23. Chlebowski R, Wactawski-Wende J, Ritenbaugh C, Hubbell A, Ascensao J, Rodabough R, et al; Women's Health Initiative Investigators. Estrogen plus progestin and colorrectal cancer in posmenopausal women. N Engl J Med 2004;350:991-1004.

24. Cauley JA, Norton L, Lippman ME, Eckert S, Krueger KA, Purdie DW, et al. Continued breast cancer risk reduction in posmenopausal women treated with raloxifene: 4-year results from the MORE trial. Multiple outcomes of raloxifene evaluation. Breast Cancer Res Treat 2001;65:125-34. 
25. The GUSTO investigators. An international randomized trial comparing four thrombolityc strategies for acute miocardial infarction. N Engl J Med 1993;329:673-82.

26. Silverstein F, Faich G, Goldstein JL, Simon LS, Pincus T, Whelton A, et al. Gastrointestinal toxicity with celecoxib vs nonsteroidal anti-inflammatory drugs for osteoarthritis and rheumatoid arthritis: the CLASS study: a randomized controled trial. JAMA 2000;284:1247-55.

27. http://www.channing.harvard.edu/nhs/questionnaires/ qx_all.shtml. Acceso el 23 de octubre de 2004

28. Grodstein F, Stampfer MJ, Manson JE, Colditz GA, Willett WC, Rosner B, et al. Postmenopausal estrogen and progestin use and the risk of cardiovascular disease. N Engl J Med 1996;335:453-61.

29. Grodstein F, Stampfer MJ, Colditz GA, Willett WC, Manson JE, Joffe M, et al. Postrmenopausal hormone therapy and mortality. N Engl J Med 1997; 336:1769-75.

30. Grodstein F, Martinez ME, Platz EA, Giovannucci E, Colditz GA, Kautzky M, et al. Postmenopausal hormone use and risk for cancer and adenoma. Ann Intern Med 1998;128:705-12.

31. Grodstein F, Manson JE, Stampfer MJ. Postmenopausal hormone use and secondary prevention of coronary events in the nurses' health study. Ann Intern Med 2001;135:1-8.

32. Cherry N, Gilmour K, Hannaford P, Heagerty A, Khan MA, Kitchener H; ESPRIT team. Oestrogen therapy for prevention of reinfarction in postmenopausal women: a randomized placebo controlled trial. Lancet 2002;360:2001-8.

33. Herrington DM, Reboussin DM, Brosnihan KB, Sharp PC, Shumaker SA, Snyder TE, et al. Effects of estrogen replacement on the progression of coronary artery atheroesclerosis. N Engl J Med 2000;343:522-9.

34. Viscoli C, Brass LM, Kernan WN, Sarrel PM, Suissa S, Horwitz RI. A clinical trial of estrogen-replacement therapy after ischemic stroke. N Engl J Med 2001;345:1243-9.

35. Moynihan R, Health I, Henry D. Selling sickness: the pharmaceutical industry and disease mongering. BMJ 2002;324:886-91.

36. Avorn J, Chen M, Hartley R. Scientific versus commercial sources of influence on the prescribing behavior of physicians. Am J Med 1982;73:4-8.

37. Orlowski JP, Wateska L. The effects of pharmaceutical frim enticements on physician prescribing pat- terns. There's no such thing as a free lunch. Chest 1992;102:270-3.

38. Moynihan R. Who pays for the pizza? Redefining the relationships between doctors and drug companies. 1: entanglement. BMJ 2003;326:1189-92.

39. Melander H, Ahlqvist-Rastad J, Meijer G, Beermann B. Evidenced based medicine--selective reporting from studies sponsored by pharmaceutical industry: review of studies in new drug applications. BMJ 2003;326:11713.

40. Rennie D. Thyroid storm. JAMA 1997;277:1238-43.

41. Moynihan R. Drug company secretly briefed medical societies on HRT. BMJ 2003;326:1161.

42. Koch K. Schering uses German medical association to promote HRT. BMJ 2003;326:1161.

43. Hays J, Ockene JK, Brunner RL, Kotchen JM, Manson JE, Petterson RE, et al; Women's Health Initiative Investigators. Effects of estrogen plus progestin on healthrelated quality of life. N Engl J Med 2003;348:183954.

44. Shumaker SA, Legault C, Rapp SR, Thal L, Wallace $\mathrm{RB}$, Ockene JK, et al. Estrogen plus progestin and the incidence of dementia and mild cognitive impairment in posmenopausal women: the Women's Health Initiative Memory Study: a randomized controled trial. JAMA 2003;289:2651-62.

45. Rapp SR, Espeland MA, Shumaker SA, Henderson VW, Brunner RL, Manson JE, et al. Effect of estrogen plus progestin on global cognitive function in postmenopausal women: the Women's Health Initiative Memory Study: a randomized controlled trial. JAMA 2003;289:2663-72.

46. Shumaker SA, Legault C, Kuller L, Rapp SR, Thal L, Lane DS, et al. Conjugated equine estrogen and incidence of probable dementia and mild cognitive impairment in postmenopausal women. Womens' Health Initiative Memory Study. JAMA 2004;291:2947-58.

47. Espeland MA, Rapp SR, Shumaker SA, Brunner R, Manson JE, Sherwin BB, et al. Conjugated equine estrogen and global cognitive function in postmenopausal women. Womens' Health Initiative Memory Study. JAMA 2004;291:2959-68.

48. Kang JH, Weuve J, Grodstein F. Postmenopausal hormone therapy and risk of cognitive decline in communitydwelling aging women. Neurology 2004;63:101-7.

49. Zuckerman D. Hype in health reporting: "checkbook science" buys distortion of medical news. Intl J Health Serv 2003;33:383-9. 
50. Stampfer MJ, Colditz GA. Estrogen replacement therapy and coronary heart disease: a quantitative assessment of the epidemiologic evidence. Prev Med 1991;20:4763.

51. Stampfer M. Commentary: hormones and heart disease: do trials and obsrvacional studies address different questions? Int J Epidemiol 2004; 33: 454-5.

52. Fletcher SW, Colditz GA. Failure of estrogen plus progestin therapy for prevention. JAMA 2002;288:3668.

53. Grady D, Rubin SM, Petiti DB, Fox CS, Black D, Ettinger B, et al. Hormone therapy to prevent disease and prolong life in postmenopausal women. Ann Intern Med 1992;117:1016-37.

54. Grady D. Postmenopausal hormones-- therapy for symptoms only. N Engl J Med 2003;348:1835-7.

55. Petitti D. Commentary: hormone replacement therapy and coronary heart disease: four lessons. Int J Epidemiol 2004;33:461-3.
56. Hulley SB, Grady D. The WHI estrogen-alone trial - do things look any better? JAMA 2004;291:1769-71.

57. Grodstein F, Clarkson TB, Manson JE. Undersatanding the divergent data on postmenopausal hormone therapy. N Engl J Med 2003,348:645-50.

58. Naftolin F, Taylor HS, Karas R, Brinton E, Newman I, Clarkson TB, et al; Women's Health Initiative. The Women's Health Initiative could not have detected cardioprotective effects of starting hormone therapy during the menopausal transition. Fertil Steril 2004;81:1498-501.

59. Neves-E-Castro M. Menopause in crisis post-Women Health Initiative? A view based on personal clinical experience. Hum Reprod 2003;18:2512-8.

Conflicto de intereses: ninguno declarado. 